Article

\title{
Diode-Pumped High Energy and High Average Power All-Solid-State Picosecond Amplifier Systems
}

\section{Jiaxing Liu ${ }^{1}$, Wei Wang ${ }^{2}$, Zhaohua Wang ${ }^{1, *}$, Zhiguo Lv $^{3}$, Zhiyuan Zhang ${ }^{4}$ and Zhiyi Wei ${ }^{1,3, *}$}

1 Beijing National Laboratory of Condensed Matter Physics, the Institute of Physics, Chinese Academy of Science, No. 8, 3rd South Street, Zhongguancun, Haidian District, Beijing 100190, China; E-Mail: liujx@iphy.ac.cn

2 Department of Physics, Capital Normal University, 105 West Third Ring Road North, Haidian District, Beijing 100048, China; E-Mail: ww@cnu.edu.cn

3 School of Physics and Optoelectronic Engineering, Xidian University, 266 Xinglong Section of Xifeng Road, Xi'an 710126, Shanxi, China; E-Mail: zhiguohohhot@126.com

4 School of Science, China University of Mining \& Technology, Ding No. 11 Xueyuan Road, Haidian District, Beijing 100083, China; E-Mail: zzy336996@yahoo.com

* Authors to whom correspondence should be addressed; E-Mails: zhwang@iphy.ac.cn (Z.H.W.); zywei@iphy.ac.cn (Z.Y.W.); Tel./Fax: +86-10-8264-9328 (Z.H.W.); +86-10-8264-8115 (Z.Y.W.).

Academic Editor: Malte C. Kaluza

Received: 12 September 2015 / Accepted: 26 November 2015 / Published: 4 December 2015

\begin{abstract}
We present our research on the high energy picosecond laser operating at a repetition rate of $1 \mathrm{kHz}$ and the high average power picosecond laser running at $100 \mathrm{kHz}$ based on bulk Nd-doped crystals. With diode-pumped solid state (DPSS) hybrid amplifiers consisting of a picosecond oscillator, a regenerative amplifier, end-pumped single-pass amplifiers, and a side-pumped amplifier, an output energy of $64.8 \mathrm{~mJ}$ at a repetition rate of $1 \mathrm{kHz}$ was achieved. An average power of $37.5 \mathrm{~W}$ at a repetition rate of $100 \mathrm{kHz}$ pumped by continuous wave laser diodes was obtained. Compact, stable and high power DPSS laser amplifier systems with good beam qualities are excellent picosecond sources for high power optical parametric chirped pulse amplification (OPCPA) and high-efficiency laser processing.
\end{abstract}

Keywords: diode-pumped solid state laser; picosecond laser; amplifier 


\section{Introduction}

High power picosecond lasers have attracted widespread attention due to their wide application in nonlinear frequency conversion, precision processing, and bio-medicine. A picosecond laser operating at a repetition rate of a few $\mathrm{kHz}$, with both high peak power and high average power, is an excellent source for optical parametric chirped pulse amplification (OPCPA), an optical parametric oscillator (OPO), optical parametric generation (OPG), and UV light generation [1-4]. At ELI-Beamlines, two picosecond lasers with $1 \mathrm{kHz},>100 \mathrm{~mJ}$, were used to pump an OPCPA system as a part of an X-ray pump laser [5]. A laser operating at a $193 \mathrm{~nm}$ wavelength could be also realized by mixing the fourth harmonic of $1064 \mathrm{~nm}$ (266 nm) and $708 \mathrm{~nm}$, which was obtained by an OPO pumped with a picosecond $532 \mathrm{~nm}$ laser [6]. Recently, ultra-short laser pulses, especially picosecond pulses, have been widely used in the field of material processing. To achieve high processing speed and productivity, high average power picosecond lasers operating at repetition rates of hundreds of $\mathrm{kHz}$ are preferred. The demand for compact, stable, and low-cost picosecond lasers has increased rapidly. Laser diodes are preferred pumps for high power lasers. Diode-pumped solid-state lasers (DPSSLs) are smaller, more stable, and have a lower cost, thus making them more practical for more applications [7-9].

In order to achieve high output power, higher power has to be pumped into the gain medium. In this case, excessive heat will be generated in the gain medium due to a finite conversion efficiency. Thus, the key issue in a high power laser system is how to remove the heat from the gain medium effectively. In the past years, several designs of high power picosecond lasers have been studied. Using rod materials, pulses with $1.5 \mathrm{~J}$ energy and 110 ps duration at $10 \mathrm{~Hz}$ [10], with $130 \mathrm{~mJ}$ and $64 \mathrm{ps}$ at $300 \mathrm{~Hz}$ [11], with $80 \mathrm{~mJ}$ and $50 \mathrm{ps}$ at $1 \mathrm{kHz}$, and with $145 \mathrm{~W}$ average power and $200 \mathrm{ps}$ duration at $3 \mathrm{kHz}$ were demonstrated with Nd-doped $\mathrm{YVO}_{4}$ and YAG [12,13]; pulses with $1 \mathrm{~J}$ and $5 \mathrm{ps}$ at $100 \mathrm{~Hz}$ [4], with $115 \mathrm{~mJ}$ before compressing at $200 \mathrm{~Hz}$ [14], and with $58.5 \mathrm{~mJ}$ at $1 \mathrm{kHz}$ were also recently reported with cryogenically cooled bulk Yb:YAG [9]. Using thin-disk technology, a $300 \mathrm{~W}$ picosecond laser operating at $10 \mathrm{kHz}$ was demonstrated with $1.6 \mathrm{ps}$ in 2013 , and a $1.3 \mathrm{~kW}$ thin-disk multi-pass amplifier running at $300 \mathrm{kHz}$ with sub-8 ps was reported in $2014[15,16]$. Recently, the team at ELI-Beamlines presented a $110 \mathrm{~mJ}$ picosecond thin-disk amplifier with good beam quality. A 1-kHz-repetition-rate thin-disk regenerative amplifier was reported with a pulse energy of $10 \mathrm{~mJ}$ and a pulse duration of $1.29 \mathrm{ps}$ after compression [5,17]. With innoslab and fiber materials, an average power of $250 \mathrm{~W}$ at $12.5 \mathrm{kHz}$ was obtained, and $97 \mathrm{~W}$ average output power at $5.47 \mathrm{MHz}$ picosecond pulses was generated from a fiber master oscillator power amplifier (MOPA) system, respectively [18,19]. Combining some of the different technologies mentioned above, a hybrid CPA laser system was reported recently. The system consisting of fiber pre-amplifiers, a bulk material-based regenerative amplifier, and multi-pass amplifiers provided $70 \mathrm{~mJ}$ pulse energy at the repetition rate of $1 \mathrm{kHz}$ and about 6 ps pulse duration after compression [20]. Although thin-disk and innoslab lasers have the advantage of easy heat dissipation, lasers based on bulk materials generally could have higher efficiencies and simpler structures, making them more attractive.

In this paper, the research on high energy and high repetition rate DSPP picosecond amplifier systems with bulk Nd-doped crystals is reported. Nd-doped laser media with large emission cross-sections and long upper level lifetimes are very suitable for laser amplifications. Due to its comparatively short upper state lifetime and broad pumping bandwidth, $\mathrm{Nd}: \mathrm{YVO}_{4}$ is appropriate for efficient oscillators and amplifiers. In addition, the $0.8 \mathrm{~nm}$ emission bandwidth of $\mathrm{Nd}: \mathrm{YVO}_{4}$ supports sub-10 or sub-100 ps 
pulses [21]. In 2010, an output power of $53 \mathrm{~W}$ with a peak power of $40 \mathrm{~kW}$ from a hybrid amplifier laser system using Nd:YVO 4 was reported, corresponding to an optical efficiency of 42.4\% [22]. In 2012, using gratings to stretch and compress the pulses, a multi-pulse picosecond laser with $14 \mathrm{~mJ}$ and a pulse duration of $28 \mathrm{ps}$ operating at a repetition rate of $1 \mathrm{kHz}$ from a $\mathrm{Nd}: \mathrm{YVO}_{4}$ regenerative amplifier was reported [23]. In 2013, a laser beam with an average power of $4.7 \mathrm{~W}$ running at the repetition rate from $1 \mathrm{kHz}$ to $10 \mathrm{kHz}$ was delivered from a diode dual-end-pumped $\mathrm{Nd}: \mathrm{YVO}_{4}$ regenerative anplifier [24]. Most recently, $10.5 \mathrm{~W}, 14.2 \mathrm{ps}$ pulses at $1064 \mathrm{~nm}$ with a repetition rate of $10 \mathrm{kHz}$ from a Nd:YVO 4 amplifier system was obtained [25] and an average power of $44.5 \mathrm{~W}$, a pulse duration of $8.8 \mathrm{ps}$ at $100 \mathrm{MHz}$ with an optical efficiency of 56\% was achieved from a sapphire face-cooled $\mathrm{Nd}$ : $\mathrm{YVO}_{4}$ slab amplifier [26]. However, $\mathrm{Nd}: \mathrm{YVO}_{4}$ has low mechanical fracture so the pump intensity must be kept under a threshold. Considering the characteristics of the $\mathrm{Nd}: \mathrm{YVO}_{4}$ crystal, we employed quasi-continuous wave laser diodes as end-pumping sources for $\mathrm{Nd}: \mathrm{YVO}_{4}$ crystals and a side-pumped $\mathrm{Nd}$ :YAG module as a power amplifier. At a repetition rate of $1 \mathrm{kHz}$, an output energy of $64.8 \mathrm{~mJ}$ centered at $1064.4 \mathrm{~nm}$ was achieved. An average power of $37.5 \mathrm{~W}$ at a repetition rate of $100 \mathrm{kHz}$ pumped by continuous wave (CW) laser diodes was obtained. Compact, stable, and high power DPSS laser amplifier systems with good beam qualities are excellent picosecond sources for high power OPCPA and high-efficiency laser processing.

\section{Experiments and Results}

\subsection{Picosecond Amplifier System at $1 \mathrm{kHz}$}

The picosecond laser system consisted of a picosecond oscillator, a regenerative amplifier, four stages of end-pumped single-pass amplifiers, and a side-pumped amplifier. The whole system was water-cooled and the timing was controlled by a homemade delay generator for synchronization. The generator had seven output channels and each channel provided a control signal at a repetition rate of $1 \mathrm{kHz}$ with delay adjustable from $0.25 \mathrm{~ns}$ to $1 \mathrm{~ms}$ for the pump lasers and Pockels cells.

\subsubsection{Picosecond Oscillator}

The seed pulse was provided by a diode-pumped picosecond oscillator designed as shown in Figure 1 . The laser crystal was an a-cut, 0.5 at. \% Nd-doped $\mathrm{YVO}_{4}$ crystal with a size of $3 \times 3 \times 5 \mathrm{~mm}^{3}$. The surfaces of the crystal were antireflection-coated at $808 \mathrm{~nm}$ and around $1 \mu \mathrm{m}(T>99 \%)$. For heat dissipation, the crystal was wrapped with indium foil and mounted tightly in a water-cooled copper heat sink, where the water temperature was maintained at $18{ }^{\circ} \mathrm{C}$. The pump was a fiber-coupled diode laser with a $50 \mu \mathrm{m}$ core diameter (BWT, Beijing, China) and $2 \mathrm{~W}$ output power at $808 \mathrm{~nm}$. With a semiconductor saturable absorption mirror (SESAM), the oscillator ran in a CW mode-locking state and provided about $400 \mathrm{~mW}$ average power at a repetition rate of $80 \mathrm{MHz}$ as shown in Figure 2a. The stability of the output power was measured to be $<0.7 \%$ rms as shown in Figure $2 \mathrm{~b}$. By controlling the dispersion of the cavity with Gires-Tournois interferometer (GTI) mirrors, the pulse duration could be changed from 10 ps to 15 ps. Figure 3 shows the intensity autocorrelation traces and spectra (AQ6315A, Ando Inc., Tokyo, Japan) of the mode-locked pulses with 10 ps and 15 ps, if $\operatorname{sech}^{2}$-pulse shapes were assumed, measured by a commercial intensity autocorrelator (Femtochrome, FR-103MN). Considering the amplification efficiency and to avoid damaging optics, a 15 ps pulse was used as the seed for the 
regenerative amplifier. The oscillator worked at room temperature without cooling for its high efficiency and low pump power.

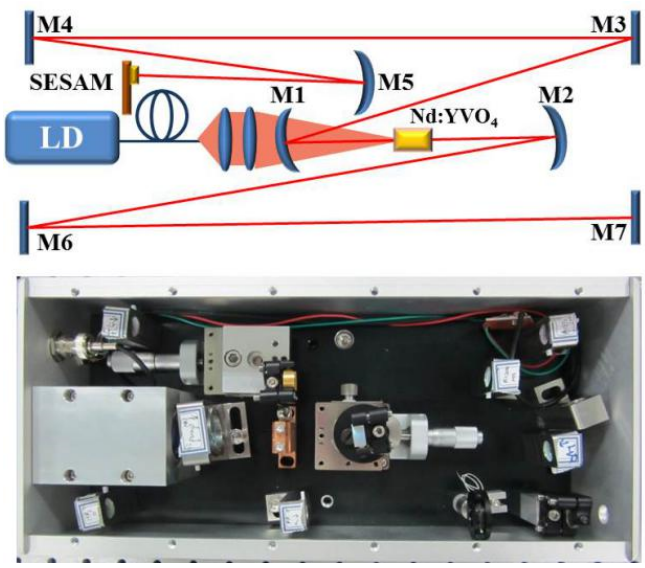

Figure 1. Set-up diagram and photograph of the picosecond oscillator. M1, M2: plane-concave pump mirrors, $R=75 \mathrm{~mm}$; M3, M4, M6: high-reflection plane mirrors; M5: concave mirror, $R=200 \mathrm{~mm} ; \mathrm{M} 7:$ output coupler, $T=10 \%$.

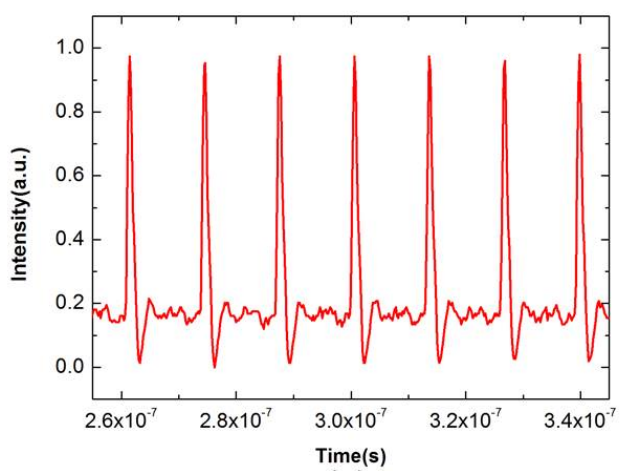

(a)

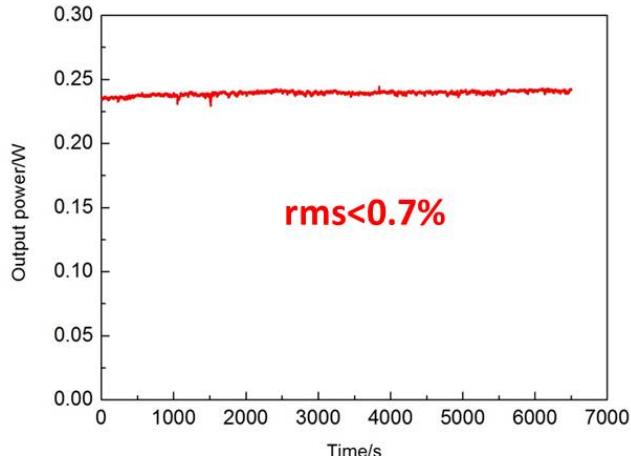

(b)

Figure 2. (a) Mode-locked pulse trains; and (b) power stability curve of the picosecond oscillator.

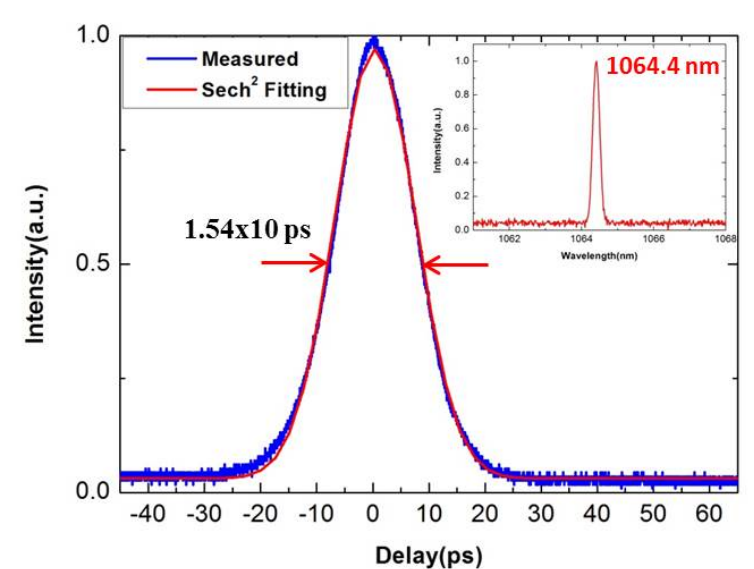

(a)

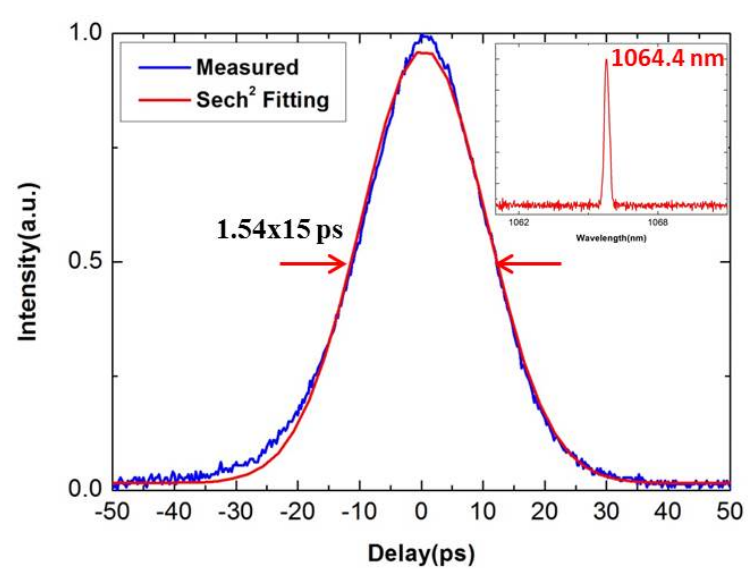

(b)

Figure 3. Intensity autocorrelation traces and spectra (insets) of the mode-locked pulses; (a) with dispersion compensation, pulse duration was $10 \mathrm{ps;}$; (b) without dispersion compensation, pulse duration was $15 \mathrm{ps}$ if $\operatorname{sech}^{2}$-pulse shapes were assumed. 


\subsubsection{Regenerative Amplifier}

The seed pulse was first amplified by the $\mathrm{Nd}: \mathrm{YVO}_{4}$ regenerative amplifier shown in Figure 4. The laser medium was a $4 \times 4 \times 10 \mathrm{~mm}^{3}$, a-cut, 0.3 at. $\% \mathrm{Nd}: \mathrm{YVO}_{4}$ crystal, which was wrapped with indium foil and mounted tightly on a water-cooled copper heat sink at $14{ }^{\circ} \mathrm{C}$. The surfaces of the crystal were antireflection-coated at $808 \mathrm{~nm}$ and around $1 \mu \mathrm{m}(T>99 \%)$. Measures were taken in the regenerative amplifier to reduce the thermal effect. Firstly, the crystal is of low doping concentration and long length. It had high absorption for the pump laser (more than 85\%) and good cooling capacity. Besides, the pump laser was a fiber-coupled quasi-CW laser diode with a $400 \mu \mathrm{m}$ core diameter centered at $808 \mathrm{~nm}$ (DILAS, Germany), which produced less heat in the crystal than a CW laser diode. The pump laser was controlled by a trigger signal with a gate width of $120 \mu \mathrm{s}$ at $1 \mathrm{kHz}$ and focused into the crystal by an imaging system with a magnification of two. Finally, a thermal compensation cavity was constructed as shown in Figure 4. Since pulse energy in the cavity was very high, laser beams in the crystal and Pockels cell were relatively large to avoid damage. The beam diameters in the crystal and Pockels cell were about $1.2 \mathrm{~mm}$ and $1.4 \mathrm{~mm}$. The intra-cavity transverse mode between the convex mirrors did not change when the focal length of the thermal lens changed from $1000 \mathrm{~mm}$ to infinity, which was caused by the increasing pump power, for ensuring the stability of the laser cavity. The stretcher and compressor were not used since the pulse duration of 15 ps was long enough in our laser system. In order to improve the contrast of the amplified pulses, the seed pulses passed through a Pockels cell to reduce the repetition rate from $80 \mathrm{MHz}$ to $1 \mathrm{kHz}$ before being injected into the amplifier. The seed pulse with about $1 \mathrm{~nJ}$ energy centered at $1064.4 \mathrm{~nm}$ was then amplified to $1.5 \mathrm{~mJ}$ at $1 \mathrm{kHz}$ under the pump energy of $8 \mathrm{~mJ}$ in the regenerative amplifier. Figure 5 shows the pulse building process in the regenerative amplifier. Figure 6 shows the beam quality of the output pulses measured by a commercial instrument (M2-200S-FW, Ophir-Spiricon Inc., North Logan, UT, USA). The result $\left(M^{2}<1.15\right)$ proved a good quality of the laser beam from the regenerative amplifier.

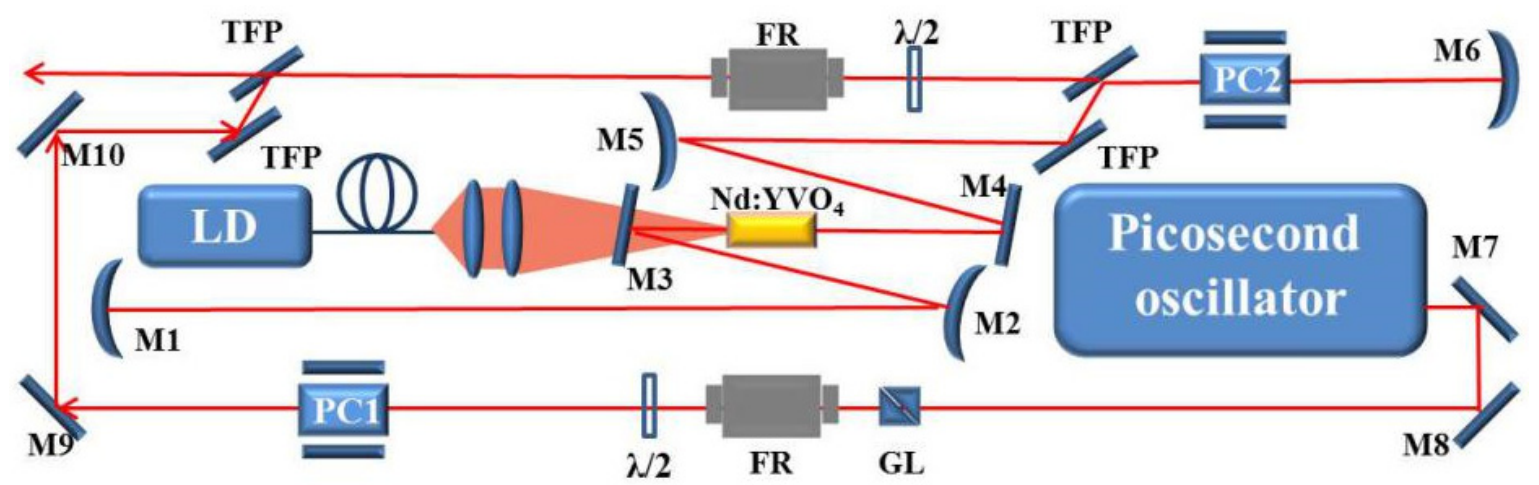

Figure 4. The scheme of the regenerative amplifier operating at a repetition rate of $1 \mathrm{kHz}$. M1, M6: Concave mirrors, $R=900 \mathrm{~mm}$; M2, M5: Convex mirrors, $R=-1000 \mathrm{~mm}$; M3, M4, M7-M10: Plane high-reflection mirrors; PC: Pockels cell; TFP: Thin-film polarizer; FR: Faraday rotator; GL: Glan prism. 


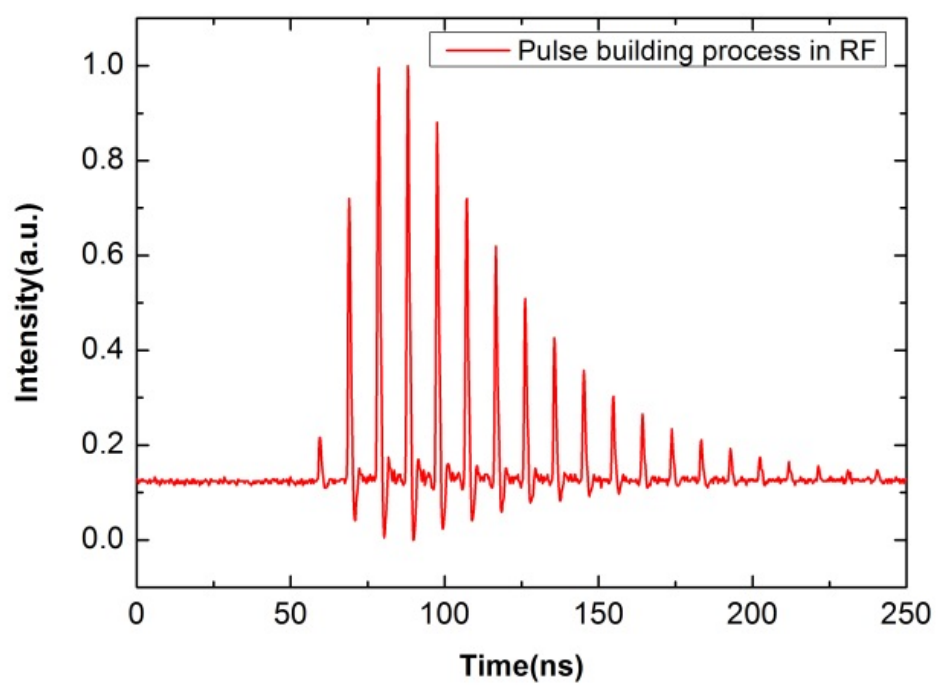

Figure 5. Pulse building process in the regenerative amplifier.

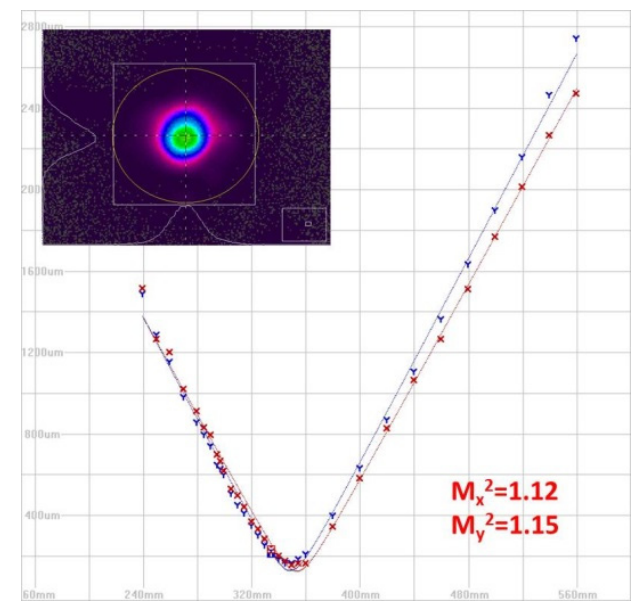

Figure 6. The beam quality of the output pulses from the regenerative amplifier.

\subsubsection{End-Pumped Single-Pass Amplifiers}

A $1.5 \mathrm{~mJ}, 1064.4 \mathrm{~nm}, 1 \mathrm{kHz}$ pulse with high contrast and good beam quality was obtained by the regenerative amplifier as described in the previous section. Higher pulse energies are required for many applications [4-6]. As previously reported [12], multiple stages of side-pumped amplifiers were used to obtain $80 \mathrm{~mJ}$ picosecond laser pulses. However, due to the inhomogeneity in the directions of the side-pumped modules, the beam quality $\left(M^{2}>4\right)$ was not good enough for some applications. To obtain a high pulse energy picosecond laser with good beam quality, we implemented a hybrid power amplifier consisting of four stages of end-pumped single-pass Nd:YVO 4 amplifiers and a side-pumped Nd:YAG amplifier as shown in Figure 7. In end-pumped amplifiers, 0.3 at. \% Nd: $\mathrm{YVO}_{4}$ crystals with a dimension of $4 \times 4 \times 10 \mathrm{~mm}^{3}$ were used as gain media and also wrapped with indium foil and mounted on water-cooled copper heat sinks at $14^{\circ} \mathrm{C}$. The fiber-coupled quasi-CW laser diodes with an $800 \mu \mathrm{m}$ core diameter centered at $808 \mathrm{~nm}$ (DILAS, Mainz-Hechtsheim, Germany) were used as pump sources, and each of them produced a $120 \mu \mathrm{s}$ pulse at $1 \mathrm{kHz}$ and focused into the crystal by an imaging system with a magnification of two. For time synchronization of the whole system, a homemade delay generator provided trigger signals at the repetition rate of $1 \mathrm{kHz}$ to drive the Pockels cells and quasi-CW pumps. 
The measured power curves and beam images are shown in Figure 8. The $1.5 \mathrm{~mJ}, 1064.4 \mathrm{~nm}, 1 \mathrm{kHz}$ picosecond pulses were amplified to be more than $34 \mathrm{~mJ}$ after four stages of end-pumped single-pass amplifiers. The total pump energy was about $125 \mathrm{~mJ}$, corresponding to an optical efficiency of $26 \%$.

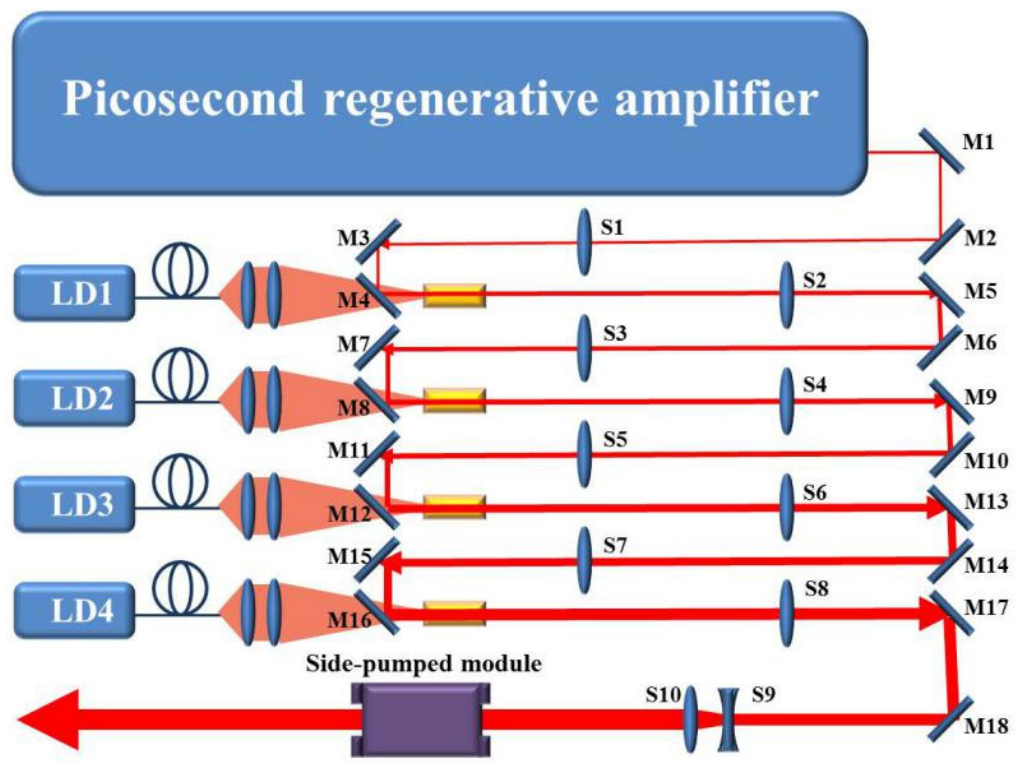

Figure 7. Diagram of the hybrid amplifier. M1-M3, M5-M7, M9-M11, M13-M15, M17-M18: Plane high-reflection mirrors; M4, M8, M12, M16: Plane high-reflection pump mirrors; S1-S8, S10: Plane-convex lens; S9: Plane-concave lens.

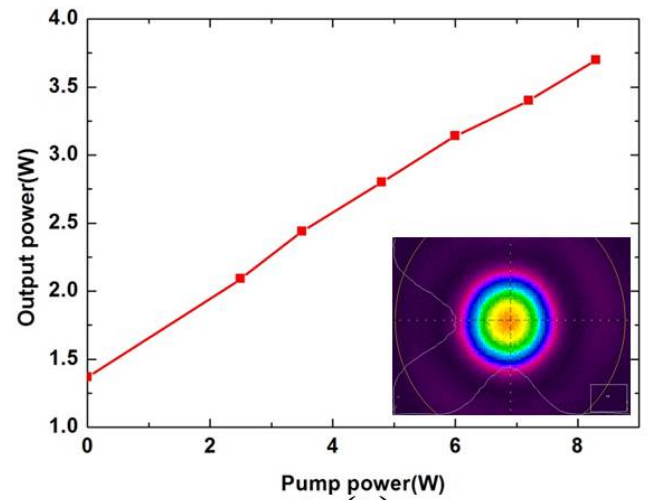

(a)

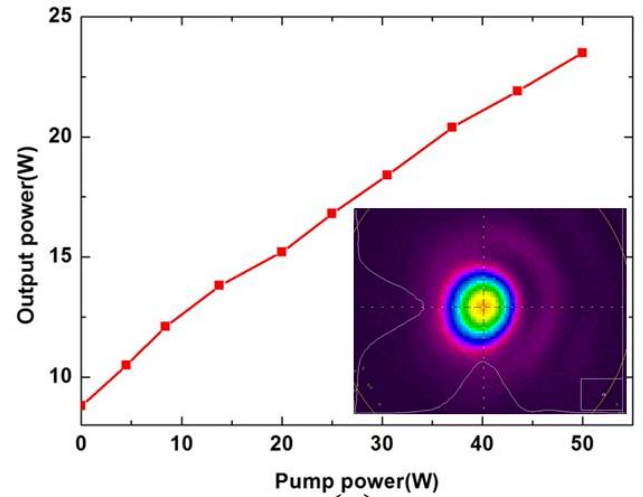

(c)

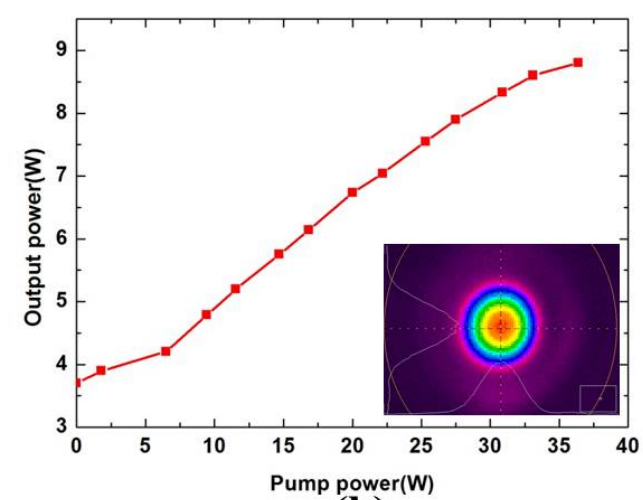

(b)

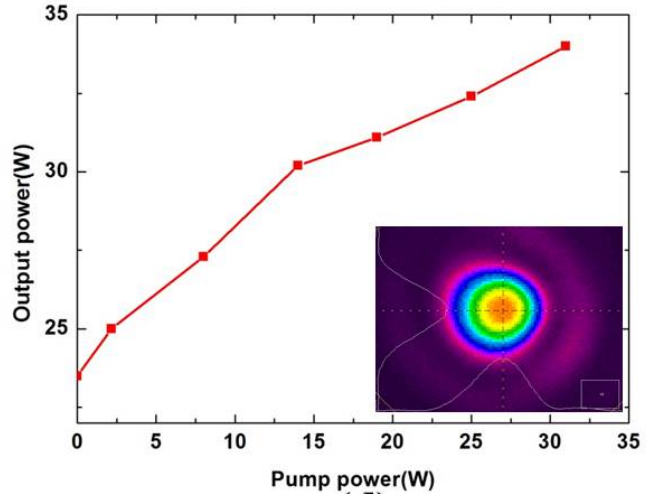

(d)

Figure 8. Power curves and beam images of each stage. (a) First stage; (b) second stage; (c) third stage; (d) fourth stage. 


\subsubsection{Side-Pumped Amplifier}

Finally, the $34 \mathrm{~mJ}$ picosecond pulses from end-pumped amplifiers were injected into the last amplifier, a module consisting of a $\Phi 8 \times 185 \mathrm{~mm}^{3} \mathrm{Nd}$ :YAG side-pumped by $\mathrm{CW}$ laser diodes. The maximum allowed pump power of $1000 \mathrm{~W}$ could be obtained at the wavelength of $808 \mathrm{~nm}$. The cooling water system maintained at $14{ }^{\circ} \mathrm{C}$ was used for the heat dissipation of the crystal. In this experiment, a pump power of $750 \mathrm{~W}$ was used and a $64.8 \mathrm{~mJ}$ picosecond laser pulse at $1 \mathrm{kHz}$ was obtained. Figure 9 shows the power curve and beam quality, where a good beam quality of $M^{2}<2$ is illustrated.

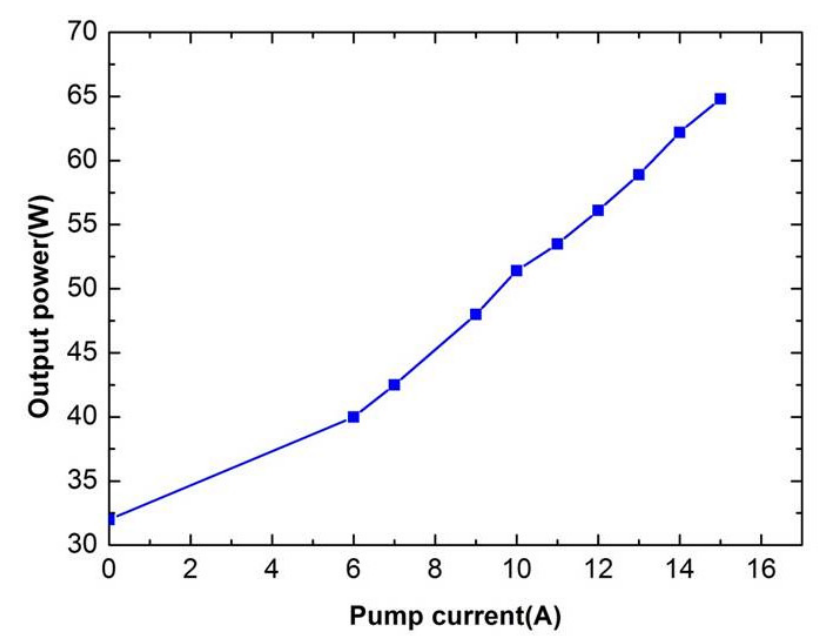

(a)

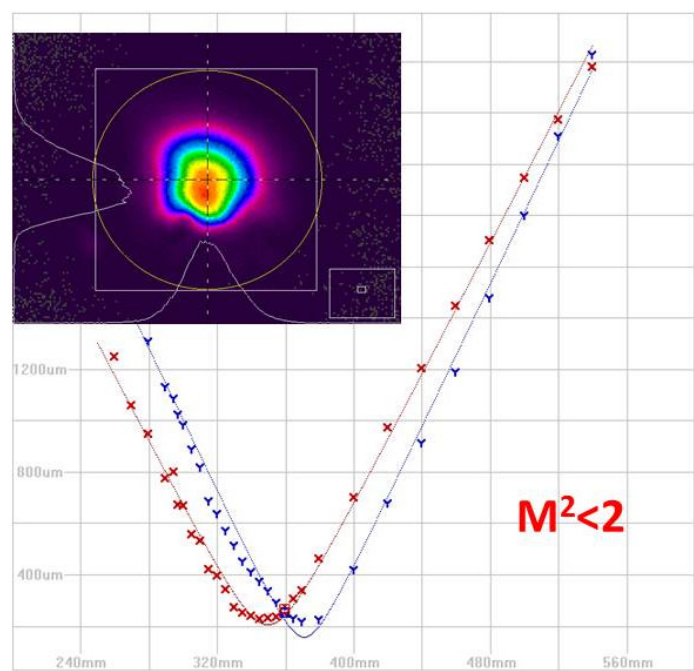

(b)

Figure 9. Power curve and beam quality of the side-pumped amplifier. (a) Power curve; (b) Beam quality.

\subsection{High Average Power Picosecond Amplifier System at $100 \mathrm{kHz}$}

As mentioned above, while the high energy picosecond laser operating at the repetition rate of $1 \mathrm{kHz}$ is an excellent pump source for OPCPA, OPO, OPG, and UV light generation, a high power picosecond laser running at a higher repetition rate, such as $100 \mathrm{kHz}$, is preferred in the field of processing. Based on the experience introduced in Section 2.1, we demonstrated a $100 \mathrm{kHz}, 37.5 \mathrm{~W}$ picosecond DPSS laser system, which was an excellent source for high efficiency laser processing.

At a higher repetition rate, the energy density is lower, but more heat is produced in the crystal. Reducing the diameter of the laser beam in the crystal is a good choice because of the smaller heat generation area and comparatively higher efficiency. Another reason for a smaller beam diameter was that a $3 \mathrm{~mm}$ diameter BBO Pockels cell was used as an electro-optic switch and worked at the repetition rate of $100 \mathrm{kHz}$, and the laser beam in the Pockels cell had to be smaller than that mentioned in Section 2.1.2. Thus, a thermal compensation cavity with a laser beam size smaller than that in Figure 4 was designed and constructed as a regenerative amplifier shown in Figure 10. The convex mirrors were replaced with concave mirrors $(R=600 \mathrm{~mm})$. The laser beam was about $550 \mu \mathrm{m}$ in the crystal and $730 \mu \mathrm{m}$ in the Pockels cell as the focal length of the thermal lens changed from $300 \mathrm{~mm}$ to infinity. This cavity was more suitable for high-repetition-rate amplifier performance. A 0.3 at. \%, a-cut, $3 \times 3 \times 10 \mathrm{~mm}^{3}$ $\mathrm{Nd}: \mathrm{YVO}_{4}$ crystal antireflection-coated at $808 \mathrm{~nm}$ and around $1 \mu \mathrm{m}(t>99 \%)$ was used for this cavity. 
The crystal was wrapped with indium foil and mounted tightly on a water-cooled copper heat sink at $14{ }^{\circ} \mathrm{C}$. The pumping source was a fiber-coupled CW laser diode with a $200 \mu \mathrm{m}$ core diameter centered at $808 \mathrm{~nm}$ (Focuslight, China). Figure 11a shows the power curve of the regenerative amplifier. At the $\mathrm{CW}$ pump power of $15.5 \mathrm{~W}, 2.6 \mathrm{~W}$ average output power was obtained at the repetition rate of $100 \mathrm{kHz}$ by injecting a $15 \mathrm{ps}$ seed pulse from a $1064.4 \mathrm{~nm}, 80 \mathrm{MHz}$ picosecond oscillator. Figure 11b shows the beam quality of the output pulses as good as $M^{2}<1.2$.

To further improve the average power, a hybrid power amplifier was also used. The hybrid power amplifier consisted of three stages of end-pumped single-pass amplifiers with $\mathrm{CW}$ laser diodes and a side-pumped module introduced in Section 2.1. The 2.6 W output pulses from the regenerative amplifier were then amplified by the single-pass amplifiers. The 0.3 at. $\%$, a-cut, $3 \times 3 \times 10 \mathrm{~mm}^{3} \mathrm{Nd}$ : $\mathrm{YVO}_{4}$ crystals were employed as gain media. At the total pump power of $88.5 \mathrm{~W}$, an output average power of $21 \mathrm{~W}$ was obtained at $1064.4 \mathrm{~nm}$, corresponding to a pulse energy of $210 \mu \mathrm{J}$ and an optical efficiency of $20.8 \%$. At last, the picosecond pulses were amplified to $37.5 \mathrm{~W}$ by the side-pumped module at a pump power of $900 \mathrm{~W}$. The pulse energy was as high as $375 \mu \mathrm{J}$ at a repetition rate of $100 \mathrm{kHz}$. Figure 12 shows the power curves and beam images of the power amplifiers.

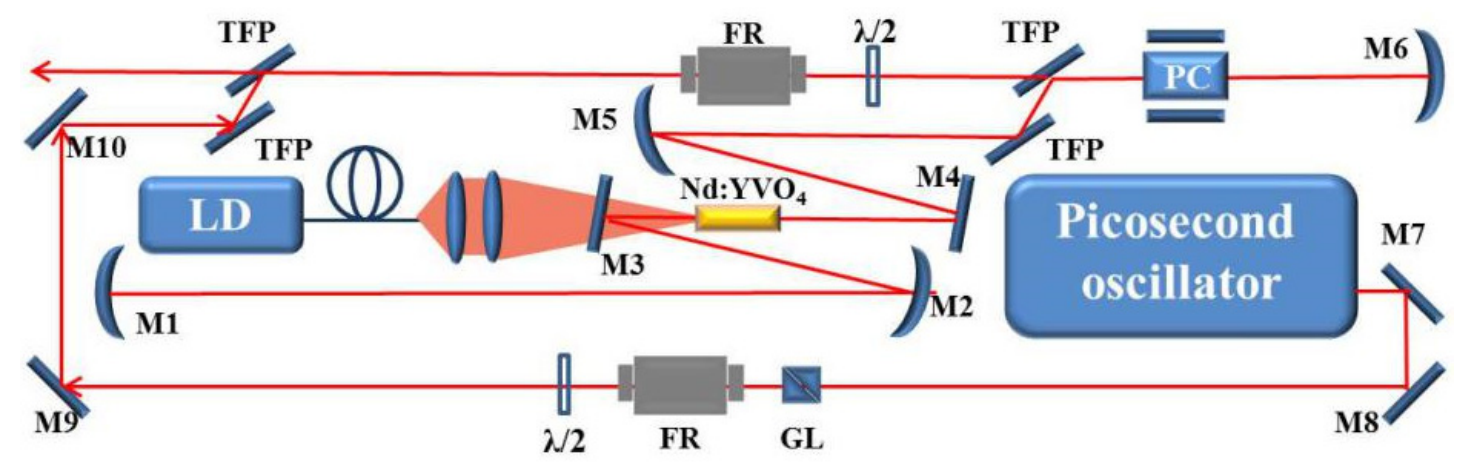

Figure 10. Diagram of the regenerative amplifier running at the repetition rate of $100 \mathrm{kHz}$. M1, M6: Concave mirrors, $R=900 \mathrm{~mm}$; M2, M5: Concave mirrors, $R=600 \mathrm{~mm}$; M3, M4, M7-M10: Plane high-reflection mirrors; PC: Pockels cell; TFP: Thin-film polarizer; FR: Faraday rotator; GL: Glan prism.

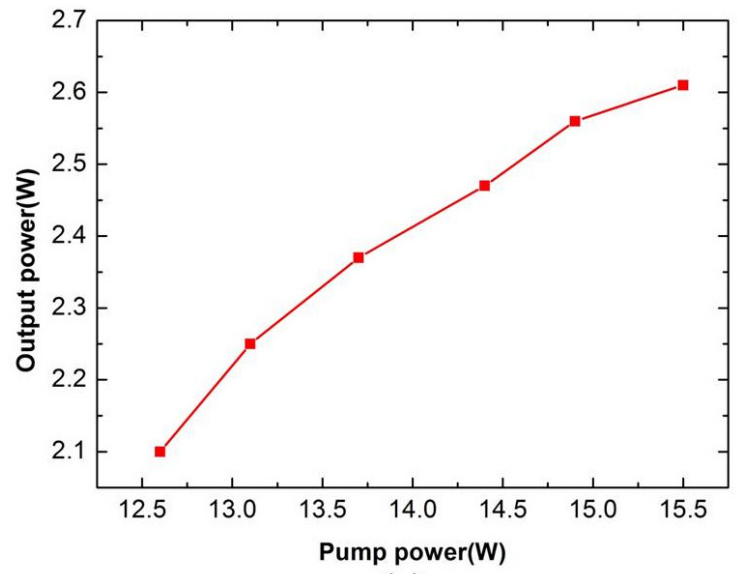

(a)

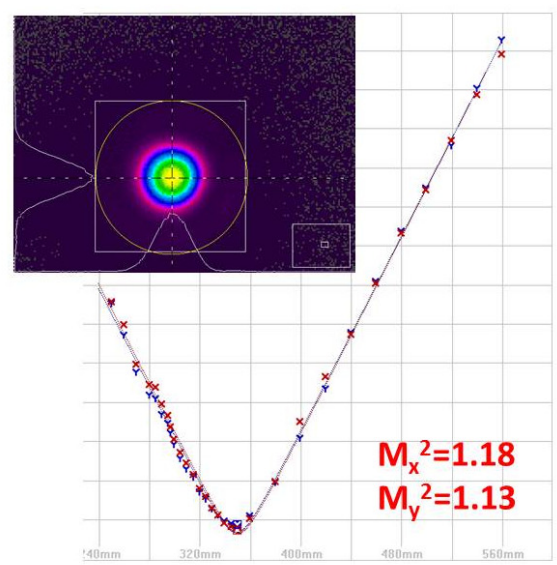

(b)

Figure 11. (a) Power curve of regenerative amplification; (b) Beam quality of the output pulses from the regenerative amplifier. 


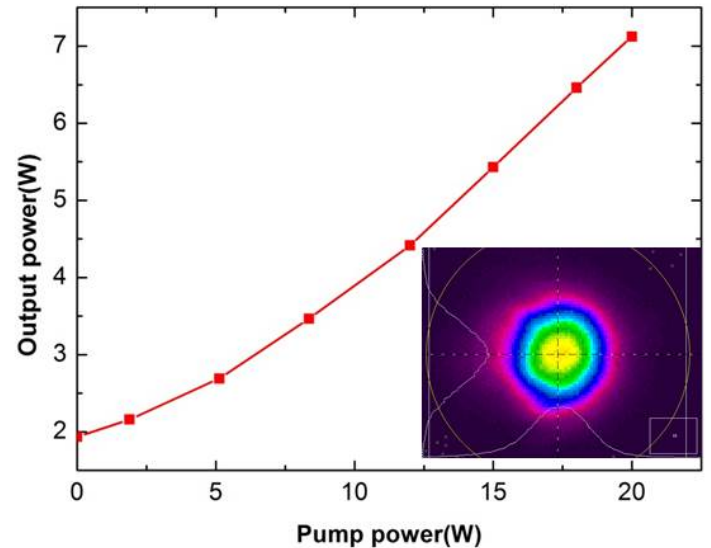

(a)

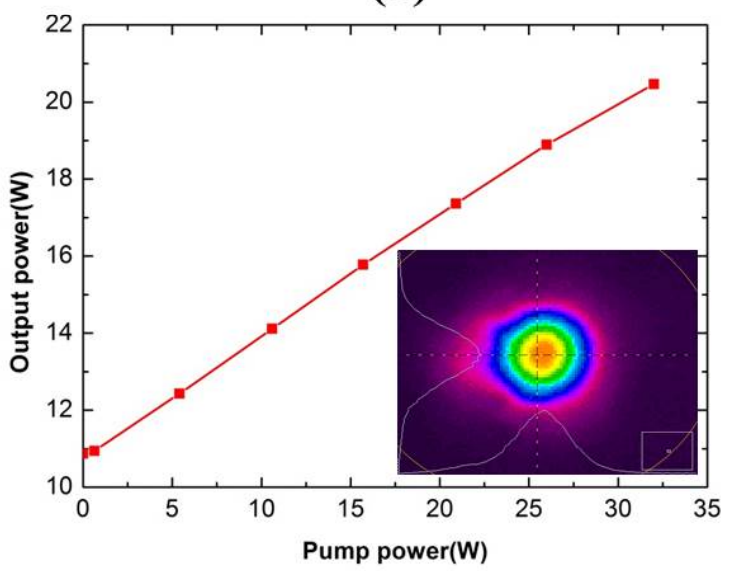

(c)

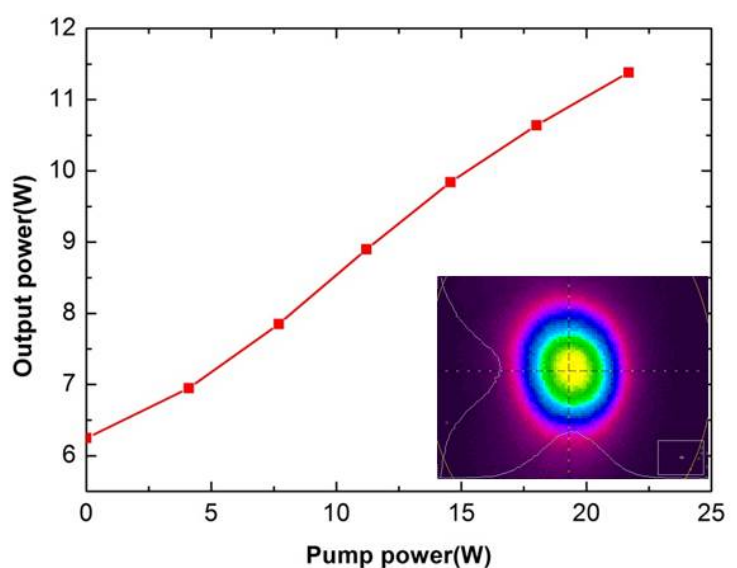

(b)

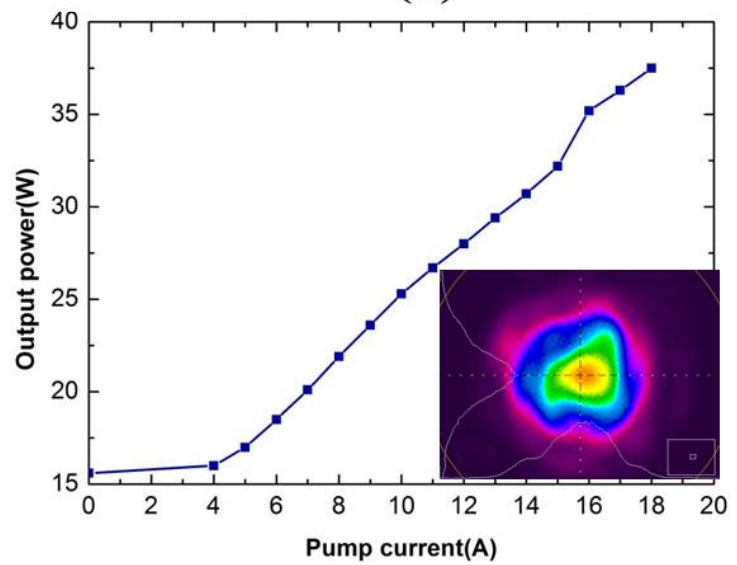

(d)

Figure 12. Power curves and beam images of each stage. (a) First stage; (b) second stage; (c) third stage; (d) side-pumped stage.

\section{Conclusions and Discussions}

In conclusion, we have introduced the latest developments in high power DPSS picosecond lasers. We presented our work on a high energy picosecond laser operating at a repetition rate of $1 \mathrm{kHz}$. and a high average power picosecond laser running at $100 \mathrm{kHz}$. With DPSS hybrid amplifiers consisting of a picosecond oscillator, a regenerative amplifier, end-pumped single-pass amplifiers, and a side-pumped amplifier, the output energy of $64.8 \mathrm{~mJ}$ at a repetition rate of $1 \mathrm{kHz}$ was achieved. An average power of $37.5 \mathrm{~W}$ at a repetition rate of $100 \mathrm{kHz}$ pumped by $\mathrm{CW}$ laser diodes was also obtained.

Although without the advantage of heat dissipation, laser systems based on bulk materials generally could have high conversion efficiency and simple structures. These characteristics make bulk materials attractive choices as laser media in high power DPSS picosecond lasers. In this paper, we demonstrated high energy and high average power picosecond laser systems with bulk Nd-doped crystals, proving them as promising candidates to achieve high power DPSS lasers.

To improve the system's capacity of heat dissipation, we took measures on the crystals, pumps, and cavity design. We used long crystals with low doping concentration and quasi-CW laser diodes as pumps. A thermal compensation cavity was designed and used as a regenerative amplifier. The crystals were water-cooled at $14^{\circ} \mathrm{C}$. In the experiments, we found that the efficiency dropped significantly when 
the pump power increased to a certain value beyond the damage threshold of the crystals. The main influence factor, we believed, was the heat accumulated in the crystals. In the next step, we will replace the water-coolers with thermal electronic coolers (TEC) or cryogenic coolers to improve the capacity of heat dissipation to achieve higher power or energy.

By the figures showing the beam qualities of the output pulses in this paper, we noticed that the beam quality deteriorated significantly after including the side-pumped amplifier despite providing a huge pump power. Its inhomogeneity in space had a great influence on the beam quality. However, the beam quality of the picosecond laser is especially important for certain applications. The side-pumped module will have to be replaced by an end-pumped amplifier for better beam quality.

\section{Acknowledgments}

This work was partially supported by the National Major Equipment Development Project of the Ministry of Science and Technology of China (2012YQ120047) and the National Natural Science Foundation of China $(11174361,61575217)$.

\section{Author Contributions}

Zhiyi Wei and Zhaohua Wang designed and supervised the research; Jiaxing Liu, Zhiguo Lv and Zhiyuan Zhang performed the research; Jiaxing Liu and Wei Wang analyzed the data; and Jiaxing Liu wrote the paper with discussions from all authors.

\section{Conflicts of Interest}

The authors declare no conflict of interest.

\section{References}

1. Ursula, K. Recent developments in compact ultrafast lasers. Nature 2003, 424, 831-838.

2. Kleinbauer, J.; Knappe, R.; Wallenstein, R. A powerful diode-pumped laser source for micro-machining with ps pulses in the infrared, the visible and the ultraviolet. Appl. Phys. B 2005, 80, 315-320.

3. Kienle, F.; Chen, K.K.; Alam, S.U.; Gawith, C.B.; Mackenzie, J.I.; Hanna, D.C.; Richardson, D.J.; Shepherd, D.P. High-power, variable repetition rate, picosecond optical parametric oscillator pumped by an amplified gain-switched diode. Opt. Express 2010, 18, 7602-7610.

4. Brendan, A.R.; Cory, B.; Keith, W.; Herman, B.; Mark, W.; Alden, C.; Federico, J.F.; Bradley, M.L.; Dinesh, P.; Carmen, S.M.; et al. 1 Joule, $100 \mathrm{~Hz}$ repetition rate, picosecond CPA laser for driving high average power soft X-ray lasers. In Proceedings of the 2014 Conference on Lasers and Electro-Optics (CLEO), San Jose, CA, USA, 8-13 June 2014.

5. Jakub, N.; Pavel, B.; Jonathan, T.G.; Zbynek, H.; Bedrich, R. $100 \mathrm{~mJ}$ thin disk regenerative amplifier at $1 \mathrm{kHz}$ as a pump for picosecond OPCPA. In Proceedings of the 2015 Conference on Lasers and Electro-Optics (CLEO), San Jose, CA, USA, 10-15 May 2015. 
6. Sadovskii, S.P.; Chizhov, P.A.; Bukin, V.V.; Brendel, V.M.; Dolmatov, T.V.; Polivanov, Y.N.; Orlov, S.N.; Garnov, S.V.; Vartapetov, S.K. Picosecond laser system with a wavelength of $193 \mathrm{~nm}$ based on a solid-state Nd:YAG laser, parametric oscillator, and ArF amplifier. Phys. Wave Phenom. 2014, 22, 223-226.

7. Rudiger, M.; Michael, K.; Christian, G.; Klaus, K.; Wilfried, P.; Ulrich, T.S.; Joachim, W. Laser processing of gallium nitride-based light-emitting diodes with ultraviolet picosecond laser pulses. Opt. Eng. 2012, 51, doi:10.1117/1.OE.51.11.114301.

8. Bai, Z.X.; Ai, Q.K.; Duan, J.P.; Chen, M.; Li, G. The research on the micro processing used all-solid-state picosecond laser. Proc. SPIE 2012, 8334, doi:10.1117/12.946044.

9. Chang, C.L.; Krogen, P.; Liang, H.K.; Stein, G.J.; Moses, J.; Lai, C.J.; Siqueira, J.P.; Zapata, L.E.; Hong, K.H. Multi-mJ, kHz, ps deep-ultraviolet source. Opt. Lett. 2015, 40, 665-668.

10. Daniel, H.; Laszlo, V.; Raphael, T.; Franz, T.; Karl, S.; Vladimir, P.; Ferenc, K. Generation of sub-three-cycle, $16 \mathrm{TW}$ light pulses by using noncollinear optical parametric chirped-pulse amplification. Opt. Lett. 2009, 34, 2459-2461.

11. Daniel, W.E.N.; Stefan, W.; Jonas, M.; Robert, K.A.; Kjeld, S.E.E. High-energy, high-repetition-rate picosecond pulses from a quasi-CW diode-pumped Nd:YAG system. Opt. Lett. 2013, 38, 3021-3023.

12. Michailovas, K.; Smilgevicius, V.; Michailovas, A. High average power effective pump source at $1 \mathrm{kHz}$ repetition rate for OPCPA system. Lith. J. Phys. 2014, 54, 150-154.

13. Nathan, B.; Benjamin, W.; Michael, C.; Lawrence, S.; Martin, R. 145 W, 3 kHz picosecond amplifier for OPCPA pumping. In Proceedings of the 2015 Conference on Lasers and Electro-Optics (CLEO): STu4O.5, San Jose, CA, USA, 10-15 May 2015.

14. Hemmer, M.; Reichert, F.; Zapata, K.; Smrz, M.; Calendron, A.R.; Cankaya, H.; Hong, K.H.; Kartner, F.; Zapata, L. Picosecond, $115 \mathrm{~mJ}$ energy, $200 \mathrm{~Hz}$ repetition rate cryogenic Yb:YAG bulk-amplifier. In Proceedings of the 2015 Conference on Lasers and Electro-Optics (CLEO): STu4O.3, San Jose, CA, USA, 10-15 May 2015.

15. Catherine, Y.T.; Marcel, S.; Robert, B.; Matthias, H.; Stephan, P.; Dirk, S.; Thomas, M. $300 \mathrm{~W}$ picosecond thin-disk regenerative amplifier at $10 \mathrm{kHz}$ repetition rate. In proceedings of the 2013 Advanced Solid-State Lasers Congress Postdeadline: JTh5A.1, Paris, France, 27 October-1 November 2013.

16. Jan-Philipp, N.; Andreas, V.; Marwan, A.A.; Dominik, B.; Dirk, S.; Alexander, K.; Thomas, G. $1.3 \mathrm{~kW}$ average output power $\mathrm{Yb}$ :YAG thin-disk multipass amplifier for multi-mJ picosecond laser pulses. In Proceedings of the 2014 Conference on Lasers and Electro-Optics (CLEO): STu1O.2, San Jose, CA, USA, 8-13 June 2015.

17. Yoshihiro, O.; Keisuke, N.; Momoko, M.; Masaaki, T.; Fumiko, Y.; Nanase, K.; Michiaki, M.; Akira, S. Yb:YAG thin-disk chirped pulse amplification laser system for intense terahertz pulse generation. Opt. Express 2015, 23, 15057-15064.

18. Schulz, M.; Riedel, R.; Willner, A.; Mans, T.; Schnitzler, C.; Russbueldt, P.; Dolkemeyer, J.; Seise, E.; Gottschall, T.; Hadrich, S.; et al. Yb:YAG Innoslab amplifier: Efficient high repetition rate subpicosecond pumping system for optical parametric chirped pulse amplification. Opt. Lett. 2011, 36, 2456-2458. 
19. Chan, H.Y.; Alam, S.U.; Xu, L.; Bateman, J.; Richardson, D.J.; Shepherd, D.P. Compact, high-pulse-energy, high-power, picosecond master oscillator power amplifier. Opt. Express 2014, 22, 21938-21943.

20. Chang, C.L.; Krogen, P.; Hong, K.H.; Zapata, L.E.; Moses, J.; Calendron, A.L.; Liang, H.; Lai, C.J.; Stein, G.J.; Keathley, P.D.; et al. High-energy, kHz, picosecond hybrid Yb-doped chirped-pulse amplifier. Opt. Express 2015, 23, 10132-10144.

21. Zayhowski, J.J.; Harrison, J. Handbook of Photonics; Gupta, M.C., Ed.; CRC Press: Boca Raton, FL, USA, 1997; pp. 326-392.

22. Wushouer, X.; Yan, P.; Yu, H.; Liu, Q.; Fu, X.; Yan, X.; Gong, M. High peak power picosecond hybrid fiber and solid-state amplifier system. Laser Phys. Lett. 2010, 7, 644-649.

23. Bai, Z.N.; Bai, Z.X.; Yang, C.; Chen, L.Y.; Chen, M.; Li, G. High pulse energy, high repetition picosecond chirped-multi-pulse regenerative amplifier laser. Opt. Laser Technol. 2013, 46, 25-28.

24. Zhang, Z.L.; Liu, Q.; Yan, P.; Xia, P.; Gong, M.L. Laser diode end-pumped Nd:YVO4 regenerative amplifier for picosecond pulses. Chin. Phys. B 2013, 22, doi:10.1088/1674-1056/22/5/054206.

25. Peng, Z.G.; Chen, M.; Yang, C.; Chang, L.; Li, G. A cavity-dumped and regenerative amplifier system for generating high-energy, high-repetition-rate picosecond pulses. Jpn. J. Appl. Phys. 2015, 54, doi:10.7567/JJAP.54.028001.

26. Masashi, A.; Hiroki, S.; Maya, K.; Yuta, S.; Katsuhiko, M.; Takashige, O. High average power, diffraction-limited picosecond output from a sapphire face-cooled Nd:YVO4 slab amplifier. J. Opt. Soc. Am. B 2015, 32, 714-718.

(C) 2015 by the authors; licensee MDPI, Basel, Switzerland. This article is an open access article distributed under the terms and conditions of the Creative Commons Attribution license (http://creativecommons.org/licenses/by/4.0/). 